\title{
LIMITED ALLELIC DIVERSITY OF PLASMODIUM FALCIPARUM MEROZOITE SURFACE PROTEIN 1 GENE FROM POPULATIONS IN THE SOLOMON ISLANDS
}

\author{
NAOKO SAKIHAMA, HIROSHI OHMAE, BERNARD BAKOTE'E, MASATO KAWABATA, KENJI HIRAYAMA, AND \\ KAZUYUKI TANABE* \\ Laboratory of Biology, Osaka Institute of Technology, Osaka, Japan; Institute of Basic Medical Sciences, University of Tsukuba, \\ Tsukuba City, Ibaraki, Japan; Solomon Islands Medical Training and Research Institute, Honiara, Solomon Islands; International \\ Center for Medical Research, Kobe University School of Medicine, Kobe, Japan; Department of Molecular Immunogenetics, Institute \\ of Tropical Medicine, Nagasaki University, Nagasaki, Japan
}

\begin{abstract}
Meiotic recombination generates allelic diversity in the Plasmodium falciparum merozoite surface protein 1 ( $m s p 1)$ gene. In this study, we monitored recombination-based diversity of $m s p 1$ in Guadalcanal, the Solomon Islands, where malaria transmission is high. We identified 5' recombinant types, $3^{\prime}$ sequence types, and $m s p 1$ haplotypes (unique associations of $5^{\prime}$ recombinant types and $3^{\prime}$ sequence types), and compared them with those from areas of low transmission in Thailand and Vanuatu. The mean number of $5^{\prime}$ recombinant types per person (multiplicity) was lower in Guadalcanal than in Thailand. Guadalcanal populations had 6-8 mspl haplotypes; the numbers are comparable to Vanuatu but much lower than in Thailand. There were marked geographic differences in distribution of $m s p 1$ haplotypes. Linkage disequilibrium in mspl was stronger in Guadalcanal than in Thailand, suggesting limited recombination events in the Solomon Islands. We suggest that the frequency of recombination events in mspl is determined not only by transmission intensity but by the number of $m s p 1$ alleles prevalent and multiplicity of infections.
\end{abstract}

\section{INTRODUCTION}

One of the obstacles to development of effective vaccines against the human malaria parasite Plasmodium falciparum is the diversity of vaccine candidate antigens in natural parasite populations. Evidence indicates that antigen diversity limits the efficacy of acquired immunity based on strain-specific anti-parasite immunity. ${ }^{1-3}$ The diversity of antigen genes differs greatly among different areas endemic for malaria. ${ }^{4-6}$ Therefore, it is important to investigate geographic differences in the genetic diversity of P. falciparum. The merozoite surface protein 1 (MSP-1) of $P$. falciparum is a major bloodstage surface antigen. ${ }^{7}$ The gene (mspl) consists of 17 blocks according to the degree of sequence conservation among alleles. ${ }^{89}$ Sequence variation in $m s p 1$ is mainly dimorphic (two major allelic forms) in all variable blocks other than block 2, which is trimorphic (three allelic forms) (Figure 1). The block is grouped according to the presence or absence of ninebasepair repeats and the type of repeating sequences. MSP-1 contains at least two regions of protective immune target: block 2 near the $\mathrm{N}$-terminus and block 17 at the $\mathrm{C}$-terminus. In an area of Africa highly endemic for malaria, human antibodies against block 2 are associated with clinical immunity in an allelic-type-specific manner. ${ }^{10}$ Block 17 encodes a Cterminal $19-\mathrm{kD}$ polypeptide fragment that is produced by processing of MSP-1 at the merozoite surface. Antibodies against this $19-\mathrm{kD}$ fragment inhibit merozoite invasion into red blood cells, ${ }^{11,12}$ and immunization with the $19-\mathrm{kD}$ fragment confers protection against challenging infections in animals. ${ }^{13}$ Invasion-inhibiting antibodies against the 19-kD fragment have been detected in sera from individuals living in areas highly endemic for malaria. ${ }^{14,15}$

Meiotic recombination is a major genetic mechanism in the generation of allelic diversity in msp $1 .^{8,16}$ The frequency of recombination events in $P$. falciparum depends primarily on

\footnotetext{
* Address correspondence to Kazuyuki Tanabe, Laboratory of Biology, Osaka Institute of Technology, 5-16-1 Ohmiya, Asahi-ku, Osaka 535-8585, Japan. E-mail: kztanabe@ge.oit.ac.jp
}

the frequency of its transmission because $P$. falciparum undergoes meiotic recombination at the mosquito stage. We have been using recombination-based haplotypes of $m s p 1$ as a polymorphic genotype marker to study allelic diversity of mspl in parasite populations. ${ }^{17-19}$ The mspl haplotypes are defined as unique associations of $5^{\prime}$ recombinant types (blocks 2-6) and 3' sequence types (block 17). In analysis of linkage disequilibirum, the prevalence of mspl haplotypes acts as an indicator of the frequency of recombination events in parasite populations. In a high-transmission area, there is little linkage disequilibrium in the $m s p 1$ sequence, ${ }^{20}$ whereas there are moderate levels of linkage disequilibrium in mesoendemic areas such as Thailand and Vietnam. ${ }^{21}$ In contrast, there are very high levels of linkage disequilibrium in Brazil, a hypoendemic area. ${ }^{22}$ However, nearly complete linkage disequilibrium occurs in Vanuatu in the southwestern Pacific, ${ }^{19}$ where the level of malaria transmission is comparable to that of Southeast Asia, suggesting that the frequency of transmission is not the sole factor determining frequency of recombination events in msp1.

In the present study, we examined $P$. falciparum populations from Guadalcanal, the Solomon Islands, which are located between Papua New Guinea and Vanuatu. Malaria is highly endemic in the Solomon Islands, and malaria transmission is more intense than in Vanuatu. Plasmodium falciparum populations in the Solomon Islands are the most likely source of the Vanuatu populations because Vanuatu is located at the southern margin of the malarious band extending from Papua New Guinea through the Solomon Islands, and the Solomon Islands are the only neighboring country where malaria is prevalent. Therefore, it would be highly instructive to investigate the diversity of $m s p 1$ haplotypes and linkage disequilibrium in msp1 in populations from the Solomon Islands, and compare them with those of populations from Vanuatu and other geographic areas. The present results show that the diversity of $m s p 1$ haplotypes is greater in Guadalcanal than in Vanuatu, as expected. In contrast, despite high endemicity, the diversity in the Solomon Islands was relatively limited, compared with populations from Thailand, and linkage disequilibrium in mspl was greater in the Solomon Islands than 


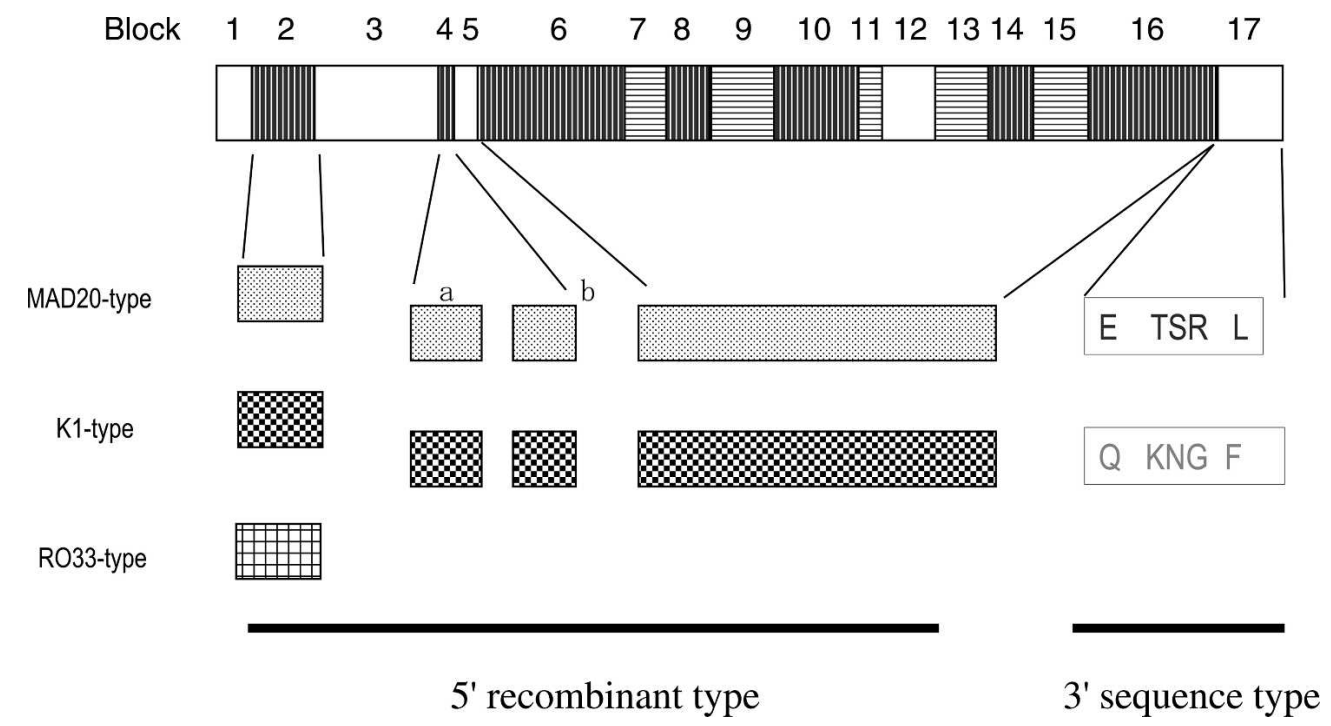

FIGURE 1. Structure of the Plasmodium falciparum merozoite surface protein 1 gene and the strategy for determination of $5^{\prime}$ recombinant type and $3^{\prime}$ sequence type. Inter-allele conserved, semi-conserved, and variable blocks are indicated by open, horizontally hatched, and vertically hatched columns, respectively. For variable blocks, MAD20-type, K1-type, and RO33-type sequences are represented by half-tone, checkerboard, and cross-striped bars, respectively. The $5^{\prime}$ recombinant type was determined by polymerase chain reaction (PCR) amplification of blocks $2-6$ using allelic type-specific primers of blocks 2 and 6, followed by a nested PCR for blocks 4a and 4b using allelic specific primers of blocks 4a and $4 \mathrm{~b}$. The five amino acid substitutions in block 17 , indicated by the one-letter codes, were determined by sequencing. The $3^{\prime}$ sequence type is the combination of those residues.

in Thailand. Also, all of our Solomon Islands isolates had the Papua New Guinea-type P. falciparum chloroquine resistance transporter (pfcrt), ${ }^{23}$ the locus that determines chloroquine resistance in $P$. falciparum.

\section{MATERIALS AND METHODS}

Study area and sample collection. Plasmodium falciparum field isolates were collected from northern Guadalcanal, the Solomon Islands, in the southwestern Pacific, from July 1995 to March 1996. Guadalcanal is the largest island in the Solomon Islands with a population of 409,000 (1999 census), and has an area of $5,336 \mathrm{~km}^{2}$, a length of $160 \mathrm{~km}$, and a width of $48 \mathrm{~km}$ (Figure 2). Malaria in Guadalcanal is hyperendemic, with parasite-positive rates of $45 \%$ and 39\% in 1994 and 1995, respectively. ${ }^{24}$ Transmission of malaria is perennial, with a peak during the rainy season from December to May. Both $P$. falciparum and $P$. vivax are prevalent, and approximately two-thirds of the parasites are $P$. falciparum.

Ninety isolates of $P$. falciparum were collected from three areas in northern Guadalcanal (Figure 2): 40 clinical isolates from outpatients of a hospital in Honiara City (area A), the capital of the Solomon Islands; 26 isolates from 3 villages (Kaotave, Tadhimboko, and Nugalitav) in area B; and 24 isolates from 1 village (Ruavatu) in area C. Area $\mathrm{C}$ is directly connected to area A by a road, whereas the three villages in area $\mathrm{B}$ are located several kilometers from the road. In rural areas $\mathrm{B}$ and $\mathrm{C}$, samples were collected from participants of malariometric surveys, and most of the donors were primary school children 8-15 years of age with a mean age of 13.2 years in area B (range $=6-59)$ and a mean age of 14.9 years in area $\mathrm{C}$ (range $=11-18)$. Parasite-positive individuals were asymptomatic in most cases in areas B and C. Approximately half of the clinical samples from Honiara were collected from subjects more than 15 years of age, with a mean age of 18.3 years (range $=4-60$ ). Giemsa-stained thick blood smears were examined microscopically, and $P$. falciparum-positive blood was withdrawn using a syringe containing EDTA and stored at $-60^{\circ} \mathrm{C}$. Parasite genomic DNA was extracted from blood using the QIAamp DNA Mini Kit (Qiagen, Hilden, Germany) according to the manufacturer's instructions. ${ }^{25} \mathrm{In}$ formed consent was obtained from the donors and the school principals. This study was reviewed and approved by the Ethical Committee of the Solomon Islands for Medical Research.

Samples collected in January $1994(\mathrm{n}=92)^{26}$ were also included in this study. These samples were from area B and included three villages (Talaura, Tadhimboko, and Kolona). The mean age of the donors was 21.6 years (range $=5-89$ ). In addition, we used clinical isolates $(\mathrm{n}=111)$ from patients who attended a malaria clinic in Mae Sot in northwestern Thailand in $1995 .^{18}$ The mean age of the donors in Thailand was 24.6 years $($ range $=13-48)$. Isolates from Vanuatu $(n=$ 164) were collected during malariometric surveys in rural villages on four islands ${ }^{19}$ : Namasari and Ontar in eastern and western Gaua island, respectively, in 1997, Big Bay in northern Santo island in 1997, Baie Barier and Pangi in eastern and western Pentecost island, respectively, in 1998, and Lingarak and Brenwe/Leviamp in eastern and northwestern Malakula island, respectively, in 1996 and 1998. Subjects were primarily school children in each village with a mean age of 6.9 years $($ range $=0-25), 12.6$ years $($ range $=2-47), 7.9$ years $($ range $=2-30), 10.6$ years $($ range $=3-36)$, and 9.8 years (range $=1-28$ ) in Gauaisland, Santo island, Pentecost island, Malakula island in 1996, and Malakula island, respectively, in 1998. The overall parasite-positive rate was $14 \%$. Plasmodium falciparum infections detected during surveys were asymptomatic in most cases.

Determination of msp1 haplotypes. The $m s p 1$ haplotypes are defined as unique associations of $5^{\prime}$ recombinant types 
a
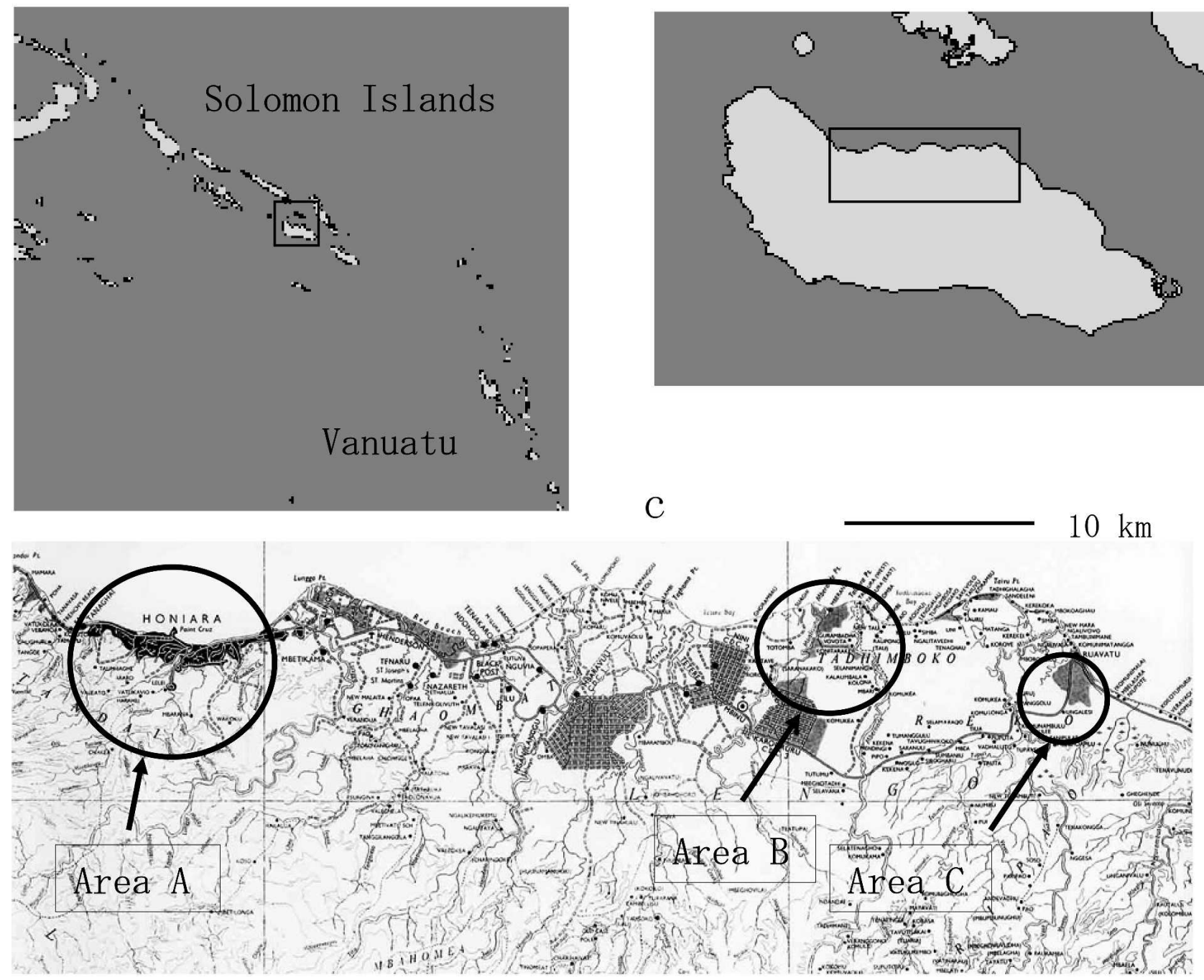

b

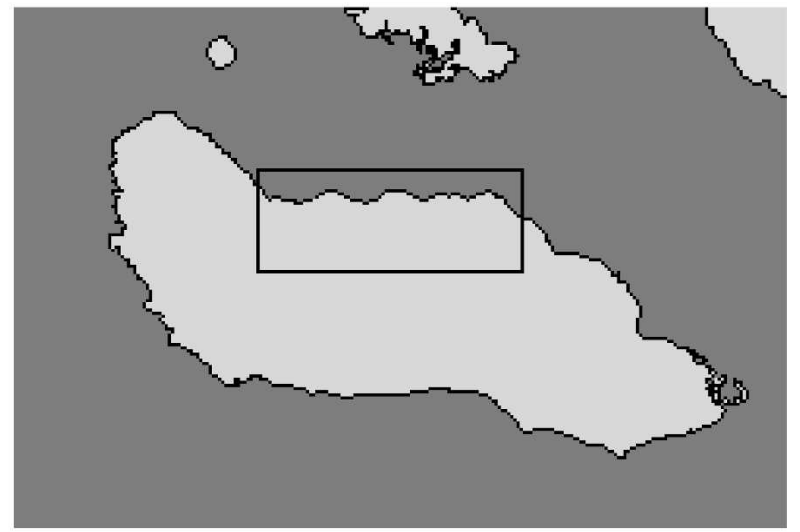

C

FIGURE 2. Location of study area. a, Solomon Islands and Vanuatu. The box shows Guadalcanal. b, Guadalcanal. The box shows the study area in northern Guadalcanal. c, The three study areas A, B and C are shown by the circles.

and $3^{\prime}$ sequence types (Figure 1). The methods used to determine $5^{\prime}$ recombinant types and $3^{\prime}$ sequence types were as follows.

The 5' recombinant types (block 2-6) of $P$. falciparum mspl (a 5-kb gene) consist of 17 distinct sequence blocks (Figure 1). Sequences of all variable blocks other than block 2 are dimorphic, either one or the other of two major allelic types: $\mathrm{K} 1$ and MAD20. Block 2 is represented by one of three major types: K1, MAD20, and RO33. The 5' recombinant types comprise unique combinations of allelic types of blocks $2,4 \mathrm{a}, 4 \mathrm{~b}$, and 6 . To date, no recombination has been found in blocks 6-16. ${ }^{18,27}$ Determination of $5^{\prime}$ recombinant types involves primary amplification of a $1-\mathrm{kb}$ region spanning blocks 2-6, followed by nested PCR amplification of block $4 a-4 b$ (approximately 100 basepairs). Procedures for determination of $5^{\prime}$ recombinant types are described elsewhere. ${ }^{26}$ For the PCR, we used $1 \mu \mathrm{L}$ of template DNA that was adjusted to be equivalent to $1 \mu \mathrm{L}$ of the original blood.

We previously reported $m s p 15^{\prime}$ recombinant types from a Thai population. ${ }^{18}$ In that study, $1 \mu \mathrm{L}$ of 20 -fold diluted DNA was used for the PCR, which is an amount not equivalent to the original volume of blood. Furthermore, we later found that the typing method used in that study was neither very sensitive nor specific, and that it produced artifacts due to the relatively weak specificity of the PCR primers used. We therefore improved that method to obtain higher sensitivity and specificity and to avoid artifacts. ${ }^{26}$ In the present study, we used the improved method to re-type $5^{\prime}$ recombinant types of the Thai samples. Although there was no significant difference in distribution of $5^{\prime}$ recombinant types between the two methods, the improved method substantially increased the rate of multiple infections of distinct $5^{\prime}$ recombinant types $(65 \%$ versus $30 \%)$ and the mean number of $5^{\prime}$ recombinant types per isolate (3.6 versus 1.8). Determination of $5^{\prime}$ recombinant types was successful in 82 of 90 isolates from the Solomon Islands samples collected in 1995 and 1996 and in 107 of 111 isolates from the Thai samples. These were compared with the $5^{\prime}$ recombinant types previously determined for Solomon Islands isolates collected in $1994(\mathrm{n}=84)^{26}$ and Vanuatu isolates in 1996-1998 $(\mathrm{n}=141) .{ }^{19}$ 
The $3^{\prime}$ sequence type (block 17), nucleotide sequence of the $3^{\prime}$ region of $P$. falciparum msp1, which encodes the C-terminal 19-kD polypeptide, was determined by direct sequencing. To obtain a clear sequence, we selected only isolates with monoinfections of a single $5^{\prime}$ recombinant type, and excluded isolates of multiple infections. The number of samples sequenced was 30 for the samples collected in 1994 from Guadalcanal, 47 for the samples collected in 1995 and 1996 from Guadalcanal, and 48 for the samples from Thailand. Because the number of monoinfections was limited in our Thai samples $(n=4)$, it was increased by diluting genomic DNA templates (20-fold). The procedures used for direct sequencing of block 17 are described elsewhere. ${ }^{19}$ The PCR products coding for amino acid residues from position 1637 to the C-terminus of MSP-1 (positions are according to Miller and others ${ }^{9}$ ) were sequenced in both directions using the BigDye Terminator Cycle Sequencing Kit version 3.1 (Applied Biosystems, Foster City, CA) and an ABI 3100 sequencer (Applied Biosystems, Foster City, CA). Sequences were verified by re-sequencing PCR products independently amplified from the same DNA. To date, five amino acid changes have been identified in block 17 in samples obtained from various geographic areas (E or Q at amino acid residue 1644 , $\mathrm{T}$ or $\mathrm{N}$ at 1691 , SR or $\mathrm{NG}$ at 1700

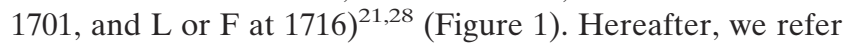
to combinations of these residues as the $3^{\prime}$ sequence type.

Sequencing of $\boldsymbol{p} \boldsymbol{f} \boldsymbol{c r t}$. Polymorphism at amino acid residues $72-76$ in the second exon of $p f c r t^{29}$ was determined for the Solomon Islands isolates with mono-infection of a 5' recombinant type from samples collected in $1994(\mathrm{n}=30)$ and samples collected in 1995 and 1996 ( $\mathrm{n}=47)$. A DNA fragment (468 basepairs) covering the second exon of pfcrt was amplified using the method used above for $m s p 1$ with primers CQF4 (5'-CAGATGGCTCACGTTTAGGTGGAGGT-3') and CQR4 (5'-TGTGTAATGTTTTATATTGGTAGGTGGA-3'), in a $50-\mu \mathrm{L}$ reaction mixture containing $1 \mu \mathrm{L}$ of template genomic DNA. The PCR product was purified and directly sequenced using CQF4 as a sequencing primer.
Statistical analyses. Frequency distributions of msp1 5' recombinant types, $3^{\prime}$ sequence types, and mspl haplotypes in different parasite populations were compared using the chisquare test with Yates' correction for data sets less than 10 and Fisher's exact test. Differences in the rate of multiple 5' recombinant type infections were tested for significance using Student's $t$-test, and differences in the number of multiple $5^{\prime}$ recombinant types per person (i.e., multiplicity) were tested for significance using a two-tailed Mann-Whitney U test. Pairs of polymorphic blocks $2,4 \mathrm{a}, 4 \mathrm{~b}$, and 6 and polymorphic sites in block 17 were subjected to an $\mathrm{R}^{2}$ test to assess linkage disequilibrium, as described elsewhere. ${ }^{21}$ Non-informative pairs (frequency $<10 \%$ in a polymorphic block or nucleotide site) were excluded from the $\mathrm{R}^{2}$ test. Significance of linkage disequilibrium was assessed using an $\mathrm{m} \times \mathrm{n}$ chi-square test and two-tailed Fisher's exact probability test. The haplotype diversity index $(h)$ of $m s p 1$ haplotypes was calculated using formula (8-5) of $\mathrm{Nei}^{30} h=\{\mathrm{n} /(\mathrm{n}-1)\} \mathrm{x}\left\{1-\Sigma \mathrm{pi}^{2}\right\}$ where $\mathrm{p}$ and $i$ are the frequency and number of $m s p 1$ haplotypes, respectively. Variance $(V)$ of $h$ was calculated using the formula $V$ $=\{2 / n(n-1)\}\left[2(n-2)\left\{\Sigma p^{3}-\left(\Sigma p^{2}\right)^{2}\right\}+\Sigma p^{2}-\left(\Sigma p^{2}\right)^{2}\right]$ that was modified from formula (8-12) of $\mathrm{Nei}^{30}$ for a haploid genome. A $P$ value less than 0.05 was considered statistically significant.

\section{RESULTS}

Diversity and multiplicity of $5^{\prime}$ recombinant types (blocks 2-6). Our typing method can potentially differentiate between 24 distinct $5^{\prime}$ recombinant types. Table 1 lists the distribution of $5^{\prime}$ recombinant types of the Solomon Islands samples collected in 1995 and 1996 and the Thai samples, together with our previously published data for Solomon Islands samples collected in $1994^{26}$ and samples from Vanuatu. ${ }^{19}$ The number of distinct $5^{\prime}$ recombinant types found in the Solomon Islands samples was $9(\mathrm{n}=6$ in 1994 and $\mathrm{n}=$

TABLE 1

Prevalence of Plasmodium falciparum merozoite surface protein 1 ( $m s p 1) 5^{\prime}$ recombinant types in the Solomon Islands in comparison with Thailand and Vanuatu*

\begin{tabular}{|c|c|c|c|c|c|c|c|}
\hline \multirow{2}{*}{$\begin{array}{c}5^{\prime} \text { recombinant } \\
\text { type }\end{array}$} & \multirow{2}{*}{$\begin{array}{c}\text { Solomon Islands }(1994) \dagger \\
\text { Area B }\end{array}$} & \multicolumn{4}{|c|}{ Solomon Islands (1995-1996) } & \multirow[b]{2}{*}{ Thailand } & \multirow[b]{2}{*}{ Vanuatu } \\
\hline & & Area A & Area B & Area C & All & & \\
\hline \#1 KKKK & 36 & 9 & 7 & 5 & 21 & 37 & 0 \\
\hline \#2 MKKK & 0 & 1 & 0 & 0 & 1 & 79 & 0 \\
\hline \#3 RKKK & 0 & 0 & 0 & 0 & 0 & 1 & 0 \\
\hline \#13 KKKM & 0 & 0 & 1 & 0 & 1 & 1 & 0 \\
\hline \#14 MKKM & 0 & 0 & 0 & 0 & 0 & 1 & 0 \\
\hline \#15 RKKM & 0 & 0 & 0 & 0 & 0 & 4 & 0 \\
\hline \#16 KMКM & 2 & 0 & 0 & 0 & 0 & 9 & 13 \\
\hline \#17 MМКM & 6 & 5 & 17 & 8 & 30 & 89 & 0 \\
\hline \#18 RMKM & 17 & 4 & 4 & 2 & 10 & 34 & 6 \\
\hline \#20 MКМM & 0 & 0 & 0 & 0 & 0 & 6 & 0 \\
\hline \#21 RKMM & 0 & 1 & 1 & 2 & 4 & 43 & 0 \\
\hline \#22 KMMM & 0 & 0 & 0 & 0 & 0 & 57 & 0 \\
\hline \#23 MMMM & 38 & 11 & 5 & 6 & 22 & 24 & 20 \\
\hline \#24 RMMM & 46 & 13 & 5 & 9 & 27 & 1 & 103 \\
\hline Total & 145 & 44 & 40 & 32 & 116 & 386 & 142 \\
\hline Number of $5^{\prime}$ recombinant types & 6 & 7 & 7 & 6 & 8 & 14 & 4 \\
\hline Rate of multiple infection (\%) & $51 / 84(60.7)$ & $8 / 35(22.9)$ & $11 / 15(44.0)$ & $10 / 22(45.5)$ & 29/82 (35.4) & 103/107 (96.3) & $1 / 141(0.7)$ \\
\hline Mean number of $5^{\prime}$ recombinant types & 1.73 & 1.26 & 1.60 & 1.40 & 1.41 & 3.6 & $1.00-1.03 \S$ \\
\hline
\end{tabular}

* 5' recombinant types are defined as associations of allelic types in msp1 blocks 2, 4a, 4b, and 6. K, M, and R denote K1, MAD20, and RO33 allelic types, respectively. Ten 5' recombinan types were not identified in any of the three geographic areas: \#4, KMKK; \#5, MMKK; \#6, RMKK; \#7, KKMK; \#8, MKMK; \#9, RKMK; \#10, KMMK; \#11, MMMK; \#12, RMMK; \#19, KKMM. $\dagger$ Sakihama and others.

$\doteqdot$ Sakihama and others. ${ }^{19}$

$\S$ Range of values separately calculated for the four islands of Vanuatu studied. 
8 in 1995-1996), which is intermediate between the numbers obtained from Thailand $(\mathrm{n}=14)$ and Vanuatu $(\mathrm{n}=4)$. Distribution of $5^{\prime}$ recombinant types differed greatly among the three geographic areas (Table 1$)\left(P<10^{-10}\right.$ for all pairs compared). These differences are due mainly to sharp contrasts in frequencies of some $5^{\prime}$ recombinant types; e.g., the frequency of type 2 was $0.205,0-0.023$ and 0 in Thailand, the Solomon Islands, and Vanuatu, respectively, and the frequency of type 24 was $0.003,0.125-0.317$, and 0.725 in Thailand, the Solomon Islands, and Vanuatu, respectively. Five 5' recombinant types (\#3, \#14, \#15, \#20, and \#22) were found only in Thailand. The prevalence of infections with multiple 5' recombinant types varied considerably among the three geographic areas (Table $1)$. The rate of multiple $5^{\prime}$ recombinant type infections in the Solomon Islands was 23-61\%, which is intermediate between Thailand (96\%) and Vanuatu (1\%). All differences between pairs of geographic areas were significant $(P<0.001)$. The mean number of $5^{\prime}$ recombinant types per person (i.e., multiplicity) in the Solomon Islands (1.4-1.7) was also intermediate between Thailand (3.6) and Vanuatu (1.0). The differences in multiplicity were highly significant $\left(P<10^{-4}\right.$ for all pairs).

In contrast to these results, distribution of $5^{\prime}$ recombinant types did not differ significantly among areas A, B and C in Guadalcanal (Table 1), but differed significantly between samples collected in 1994 and samples in 1995 and 1996 in area B $(P<0.0005)$. This difference was due mainly to temporal fluctuations of five major 5 ' recombinant types (\#1, \#17, $\# 18$, \#23, and \#24) (Table 1). A slight variation in the prevalence of multiple 5 ' recombinant type infections was observed. In the samples collected in 1995 and 1996, the rate of multiple infections and multiplicity were higher in rural areas (areas B and C) than in Honiara City: multiple infection rate $=44-46 \%$ versus $23 \%$, respectively; multiplicity $=1.4-1.6$ versus 1.26 , respectively. However, these differences were not significant. In area B, there was temporal variation in multiplicity between the samples from 1994 and the samples from 1995 and 1996 (1.7 versus 1.4, respectively), but the difference was not significant.

We examined effects of transmission season, host age, and parasite density on mspl diversity for samples $(\mathrm{n}=35)$ collected in area A (Honiara) in 1995 and 1996. The rate of multiple infection and multiplicity were slightly higher in the rainy season $(\mathrm{n}=11)$ than in the dry season $(\mathrm{n}=24)$ : multiple infection rate $=27.3 \%$ versus $20.8 \%$, respectively; multiplicity $=1.36$ versus 1.25 , respectively. However, these differences were not significant. The multiple infection rate and multiplicity did not differ significantly between subjects less than 15 years of age and those at least 15 years of age $(n=15$ and 20, respectively). There was no difference in multiple infection rate or multiplicity between a parasite density of at least 4,000 parasites/ $\mu \mathrm{L}$ and a parasite density less than 4,000 parasites/ $\mu \mathrm{L}$ ( $\mathrm{n}=17$ and 18 , respectively). Similar analysis was not performed for populations in areas $\mathrm{B}$ and $\mathrm{C}$ because of limited or biased distribution of samples among seasons, ages, and parasite densities. In samples from area B collected in January 1994 (rainy season), the multiple infection rate and multiplicity did not differ significantly between subjects less than 15 years of age and those at least 15 years old $(n=57$ and 27 , respectively).

$3^{\prime}$ sequence types (block 17). The Solomon Islands samples contained all five previously reported nucleotide polymor- phisms, ${ }^{21,28}$ and their sequence types were Q-KNG-L, QKNG-F, E-KNG-L, and E-TSR-L (Table 2). All of these sequence types were also found in both Thailand and Vanuatu. Distribution of these $3^{\prime}$ sequence types differed greatly between the Solomon Islands and other areas: $P<10^{-6}$ and $P<$ $10^{-10}$ for comparison with Thailand and Vanuatu, respectively. In Guadalcanal, there was no significant difference in distribution of $3^{\prime}$ sequence types among areas A, B, and C or between samples collected in 1994 and samples in 1995 and 1996. However, when samples were pooled to make two major groups for the Fisher's exact test, a significant difference appeared between 1994 and 1995-1996 ( $P=0.01)$.

Diversity of msp1 haplotypes. Among the Solomon Islands samples, there were six distinct msp1 haplotypes in the 1994 samples and eight distinct mspl haplotypes in the 1995-1996 samples (Table 2). These numbers are comparable to those for Vanuatu $(\mathrm{n}=6)$, but are much lower than those for Thailand $(\mathrm{n}=16)$. Haplotype diversity $(h)$ of the Solomon Islands populations (0.78-0.80) was intermediate between those of Thailand (0.89) and Vanuatu (0.43-0.68). There were marked differences in distribution of $m s p 1$ haplotypes among the three geographic areas $\left(P<10^{-10}\right)$ (Figure 3). Consistently, of 19 distinct msp1 haplotypes observed in these areas, only one haplotype (MMMM-ETSRL) was found in all areas. The Solomon Islands populations shared four haplotypes with Thailand and three haplotypes with Vanuatu. Only one haplotype was shared by Thailand and Vanuatu. These distributions suggest that the Solomon Islands populations comprise a transitional mixture between those of Thailand and Vanuatu.

In Guadalcanal, distribution of $m s p 1$ haplotypes did not significantly differ among areas A, B, and C, but differed significantly between the 1994 samples and the 1995-1996 samples. This temporal difference is due largely to fluctuations in frequencies of mspl haplotypes in the two populations (Figure 3), similar to temporal fluctuations observed for frequencies of some $5^{\prime}$ recombinant types (Table 1).

Linkage disequilibrium in msp1. We analyzed linkage disequilibrium for the Solomon Islands area A 1995-1996 samples $(n=27)$ and area B 1994 samples $(n=30)$. Of the 15 informative pairs within and between polymorphic blocks 2-6 and polymorphic nucleotides in block 17, only one pair was not significant for both the Solomon Islands 1994 samples and 1995-1996 samples (Figure 4), which indicated strong linkage disequilibrium throughout the entire mspl gene. In contrast, the Thai population $(\mathrm{n}=33)$ showed limited linkage disequilibrium: 7 of 15 pairs were not significant.

Polymorphism in p f crt. All of the Solomon Islands isolates $(\mathrm{n}=77)$ had an allele coding for SVMNT at residues 72-76 in the second exon of $p f c r t$; this is a Papua New Guinea-type chloroquine-resistant allele of $p f c r t .^{23}$ Recently, we reported the monomorphic prevalence of this resistant type in all four islands in Vanuatu, ${ }^{31}$ suggesting monomorphic prevalence of the Papua New Guinea-type chloroquine-resistant $p f c r t$ allele in the southwestern Pacific.

\section{DISCUSSION}

Several genetic mechanisms are involved in the generation of allelic diversity of $P$. falciparum genes: a point mutation causing a single nucleotide polymorphism; an insertion/ 
TABLE 2

Distribution of Plasmodium falciparum merozoite surface protein 1 ( $m s p 1)$ haplotypes in the Solomon Islands in comparison with Thailand and Vanuatu*

\begin{tabular}{|c|c|c|c|c|c|c|c|}
\hline \multirow[b]{2}{*}{$5^{\prime}$ recombinant type } & \multicolumn{5}{|c|}{$3^{\prime}$ sequence type } & \multirow{2}{*}{$\begin{array}{l}\text { No. of msp1 } \\
\text { haplotypes }\end{array}$} & \multirow[b]{2}{*}{$h \pm \mathrm{SE}$} \\
\hline & $\overline{\text { QKNGL }}$ & QKNGF & EKNGL & ETSRL & Total & & \\
\hline \multicolumn{8}{|l|}{ Solomon Islands (1994) } \\
\hline KKKK & 10 & 0 & 0 & 0 & 10 & & \\
\hline MMKM & 0 & 0 & 0 & 1 & 1 & & \\
\hline RMKM & 0 & 0 & 0 & 5 & 5 & & \\
\hline MMMM & 0 & 0 & 9 & 1 & 10 & & \\
\hline RMMM & 0 & 0 & 0 & 4 & 4 & & \\
\hline Total & 10 & 0 & 9 & 11 & 30 & 6 & $0.78 \pm 0.04$ \\
\hline \multicolumn{8}{|c|}{ Solomon Islands (1995-1996) } \\
\hline KKKK & 9 & 0 & 0 & 0 & 9 & & \\
\hline MMKM & 1 & 0 & 0 & 13 & 14 & & \\
\hline RMKM & 0 & 0 & 0 & 1 & 1 & & \\
\hline MMMM & 0 & 1 & 5 & 3 & 9 & & \\
\hline RMMM & 0 & 0 & 0 & 14 & 14 & & \\
\hline Total & 10 & 1 & 5 & 31 & 47 & 8 & $0.80 \pm 0.03$ \\
\hline \multicolumn{8}{|l|}{ Thailand } \\
\hline KKKK & 2 & 0 & 0 & 0 & 2 & & \\
\hline MKKK & 9 & 0 & 4 & 0 & 13 & & \\
\hline KMKM & 0 & 0 & 0 & 1 & 1 & & \\
\hline MMKM & 0 & 2 & 12 & 2 & 16 & & \\
\hline RMKM & 0 & 0 & 3 & 0 & 3 & & \\
\hline RKMM & 0 & 0 & 3 & $1 \dagger$ & 4 & & \\
\hline KMMM & 1 & 1 & 2 & 3 & 7 & & \\
\hline MMMM & 0 & 0 & 1 & 1 & 2 & & \\
\hline Total & 12 & 3 & 25 & 8 & 48 & 16 & $0.89 \pm 0.03$ \\
\hline \multicolumn{8}{|l|}{ Vanuatu } \\
\hline KMKM & 0 & 0 & 13 & 0 & 13 & & \\
\hline RMKM & 0 & 0 & 0 & 6 & 6 & & \\
\hline MMMM & 0 & 0 & 0 & 19 & 19 & & \\
\hline RMMM & 0 & 69 & 6 & 27 & 102 & & \\
\hline Total & 0 & 69 & 19 & 52 & 140 & 6 & $0.43 \pm 0.90-0.68 \pm 0.07 \S$ \\
\hline
\end{tabular}

deletion of repetitive sequences, resulting in a repeat-length polymorphism; and meiotic recombination that produces novel alleles in progeny that differ from the parental alleles. Repeat length polymorphism is a prominent feature of $P$. falciparum antigen genes such as $m s p 1, m s p 2$, and glurp. Accordingly, repeat sequences in those loci have been widely used as polymorphic markers for genotyping of $P$. falciparum. ${ }^{32}$ Repeat length polymorphism is probably generated by a mitotic replication slippage mechanism (slipped strand mispairing), ${ }^{33}$ and is presumed to evolve rapidly. ${ }^{26,31}$ Unequal crossing-over or gene conversion at meiosis may also be involved in the generation of repeat length polymorphism. Diversity of mspl haplotypes examined in the present study is distinct from that of repeat length polymorphisms, and is presumably generated by meiotic recombination in nonrepetitive sequences. The present method identifies recombinant types in the $5^{\prime}$ region (block 2-6), sequence types in the $3^{\prime}$ regions (block 17), and mspl haplotypes (unique associations of 5' recombinant types and 3' sequence types). Simultaneously, this method monitors the number and distribution of $5^{\prime}$ recombinant types in a parasite population, and multiplicity of infections (the number of 5' recombinant types per person). In the present study, we analyzed recombination-generated diversity of $m s p 1$ and multiplicity of infections in a hyperendemic area in Guadalcanal, the Solomon Islands, and compared them with those of mesoendemic areas in Thailand and Vanuatu. We selected these geographic areas because all analysis of samples from these areas was performed under the same experimental conditions.

The diversity of $5^{\prime}$ recombinant types and multiplicity of infections in Guadalcanal $P$. falciparum populations were lower than those of northwestern Thailand, despite the high endemicity in Guadalcanal. It is generally believed that diversity and multiplicity of infection increase as transmission frequency increases. Therefore, the present results are not consistent with this presumed correlation. A similar discrepancy has been reported in Papua New Guinea, where malaria transmission is more intense than in Thailand but the diversity of $m s p 2$ and multiplicity of infections are relatively low. ${ }^{34}$ The present results also indicate that linkage disequilibrium within mspl is greater in Guadalcanal than in Thailand. Linkage disequilibrium may derive from clustering of particular alleles. To avoid potential bias due to such clustering, we selected samples only from a local population (area A 19951996 samples and area B 1994 samples) and did not combine populations from different areas of Guadalcanal. Nevertheless, we detected strong linkage disequilibrium in $m s p 1$, indicating that limited recombination events are the most likely cause of this linkage disequilibrium. Although the annual entomologic inoculation rates (EIRs) of the present study areas were not available, previous reports indicate that areas close to area B have a very high EIR: 584-1,022 bites per person per year, ${ }^{35}$ a level comparable with those of high-transmission 


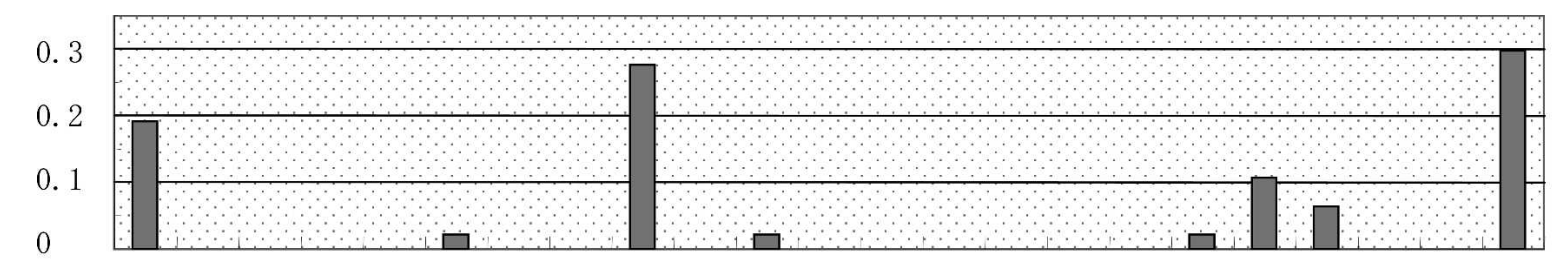

Solomon

1995-1996

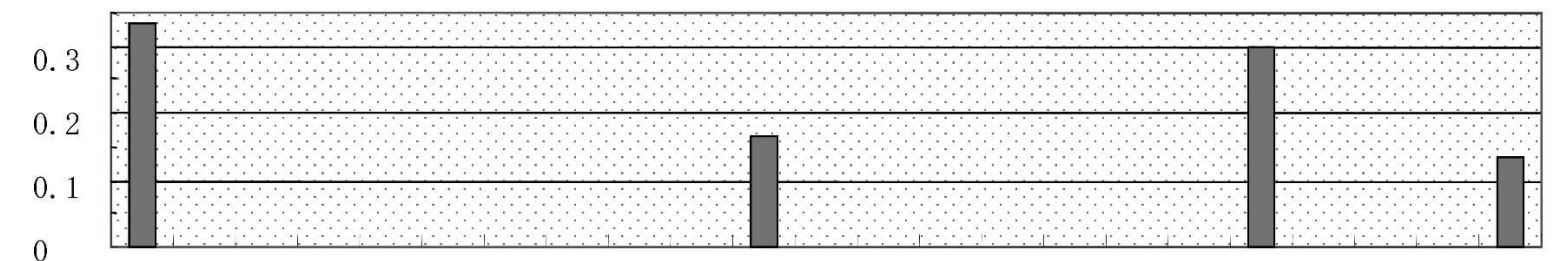

Solomon 1994

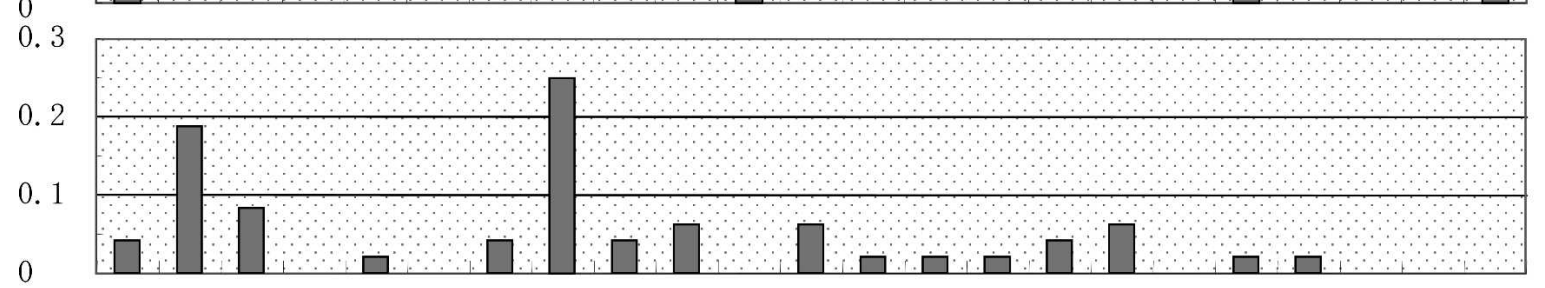

Thailand

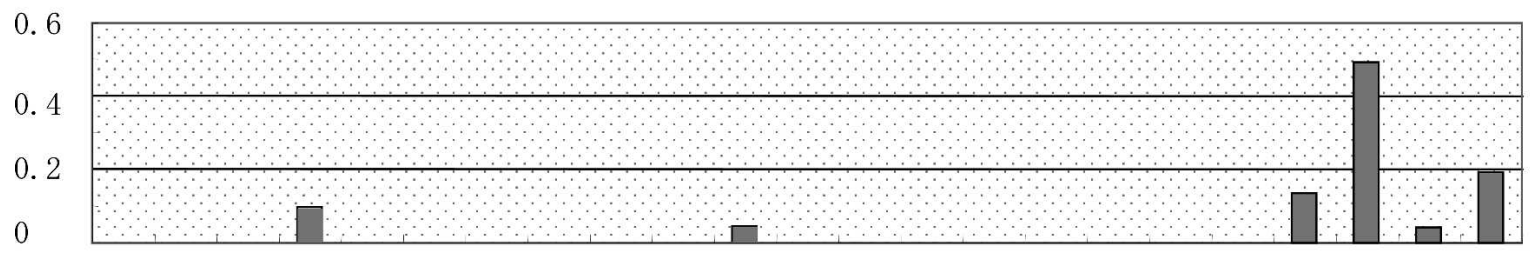

Vanuatu

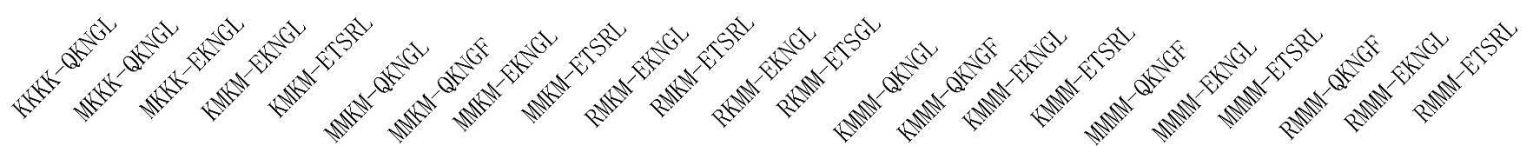

FIGURE 3. Frequency distribution of merozoite surface protein 1 ( $m s p 1)$ gene haplotypes in Plasmodium falciparum isolates from the Solomon Islands, Thailand, and Vanuatu. The $m s p 1$ haplotypes, which are unique associations of $5^{\prime}$ recombinant types and $3^{\prime}$ sequence types, are indicated along the $\mathrm{x}$-axis.

areas in Africa such as Tanzania. ${ }^{36}$ Thus, the question arises as to why linkage disequilibrium is strong in a hightransmission area. We have previously proposed that frequency of transmission is not the sole determinant of the strength of linkage disequilibrium in $m s p 1$, and that other factors are also intimately involved. ${ }^{19}$ We suggest that at least three variables are associated with linkage disequilibrium in msp1: 1) the number of alleles prevalent in a local area, 2) the rate of multiple allele infections, and 3) the number of alleles per infection (multiplicity). Effects of those variables were weak or relatively limited in the present samples from Guadalcanal, compared with the samples from Thailand. This suggests a low frequency of out-crossing, resulting in the observed linkage disequilibrium in Guadalcanal. In addition to the three variables, limited gametocyte production among the asexual parasite populations may be added: infections in Guadalcanal are in some way synchronized, so that gametocyte production at any one time may be limited to one of the asexual clones present, whereas in Thailand this is not the case.

Since epidemiologic settings vary substantially between and within geographic areas, a direct comparison of multiplicity of infection must be cautious. Multiplicity tends to decrease with age in areas highly endemic for malaria and it is lower in asymptomatic carriers than in individuals with clinical malaria. 5,37 Age-dependent acquisition of strain-specific immu- nity, which is mounted after repeated infections of different genotypes, is considered to contribute to the reduction of multiplicity. In contrast, in low-transmission areas multiplicity does not always correlate with age or the presence of clinical malaria. In the present study, isolates were collected from both symptomatic patients and asymptomatic carriers. We were unable to find a relative reduction of multiplicity of msp1 5' recombinant types in asymptomatic partiallyimmune adults in rural areas (areas B and C) in Guadalcanal because our samples from rural villages were limited in number and had biased distribution among ages (primarily school children) and parasite density. We therefore do not consider that our results exclude the presence of age-dependent reduction of multiplicity in asymptomatic semi-immune carriers in the Solomon Islands. Nevertheless, multiplicity in area A (Honiara City) was significantly lower than in areas B and C. Acquired strain-specific immunity might not be so intense to significantly reduce multiplicity in areas B and C. Low multiplicity in area A, compared with areas B and C, may simply be due to limited mosquito biting or relatively easy accessibility to anti-malarial drugs. Our Thai samples were from clinical cases, and thus are comparable with hospital samples (area A) in Guadalcanal. Multiplicity in Thailand was significantly higher than in Guadalcanal, despite a lower transmission level in Thailand. Samples from Vanuatu were from asymptomatic carriers and thus comparable to those from 


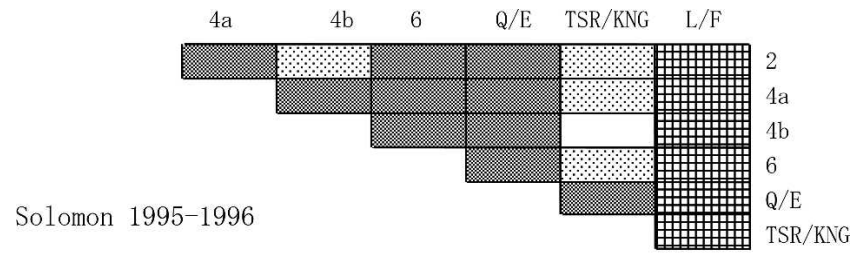

Solomon 1994

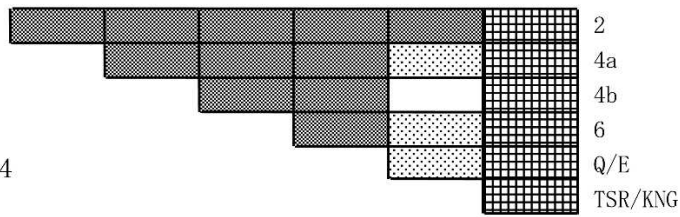

Thail and
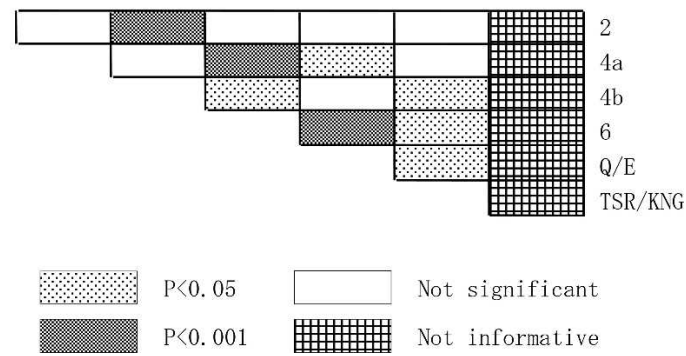

FIgURE 4. Linkage disequilibrium in the Plasmodium falciparum merozoite surface protein 1 gene in populations from the Solomon Islands (area A = 1995-1996; area B = 1994) and Thailand. Pairs of polymorphic blocks $2,4 \mathrm{a}, 4 \mathrm{~b}$, and 6 , and three polymorphic residue sites $(\mathrm{Q} / \mathrm{E}, \mathrm{TSR} / \mathrm{KNG}$, and $\mathrm{L} / \mathrm{F})$ in block 17 were subjected to the $\mathrm{R}^{2}$ test.

rural villages in Guadalcanal (areas B and C). Multiplicity in Vanuatu was nearly 1.0 and significantly lower than in Guadalcanal, where transmission is more stable and higher than in Vanuatu. These results indicate that the level of multiplicity in the Solomon Islands was intermediate between Thailand and Vanuatu, reinforcing the idea that multiplicity is not always associated with the intensity of malaria transmission.

The present study also showed temporal variation in the distribution of msp1 haplotypes in area B in Guadalcanal between the 1994 samples and the 1995-1996 samples. The present finding that all isolates from the two populations have the chloroquine-resistant $p f c r t$ alleles indicates that a population change due to chloroquine pressure is not responsible for this temporal fluctuation. A possible explanation for this fluctuation is that the area B 1994 samples and 1995-1996 samples were from different villages. However, for both the 1994 samples and 1995-1996 samples, we found no difference in distribution of mspl haplotypes among villages. Also, within a single village (Tadhimboko in area B), the distribution differed significantly between 1994 samples and 19951996 samples. Thus, there was a temporal change in distribution within area B. A previous study has shown considerable temporal variation in distribution of msp1 5' recombinant types in hypoendemic areas in Brazil. ${ }^{38}$ The temporal variation in Brazil may be due to migration of laborers. In addition to such migration, strain-specific immunity to certain mspl haplotypes may be involved in the temporal variation presently observed in the distribution in the Solomon Islands. Studies of $P$. falciparum populations from Irian Jaya suggest that strain-specific immunity is a factor in temporal variation in frequency of $m s p 2$ alleles. ${ }^{39}$
In the present study, Guadalcanal $P$. falciparum populations did not exhibit seasonal change in the distribution of msp1 5' recombinant types, the rate of multiple infections, or multiplicity of infections. A clear seasonal variation in the diversity of antigen genes and multiplicity has been observed in low-transmission areas such as Sudan, ${ }^{40}$ where transmission ceases nearly completely during the dry season. In the Solomon Islands, transmission of malaria is perennial, although it decreases during the dry season. Therefore, a direct comparison of seasonal changes in transmission cannot be made between the Solomon Islands and Sudan. In a perennial transmission area in Benin, reduced transmission had no substantial influence on the diversity of $m s p 2$ alleles or multiplicity of infections. ${ }^{41}$ Thus, seasonal changes in malaria transmission do not always affect the diversity of $m s p 1$ alleles and multiplicity of infections in area with relatively high transmission.

The present study is the first to document the prevalence of particular pfcrt alleles in the Solomon Islands. Plasmodium falciparum populations in northern Guadalcanal exhibited monomorphic prevalence of a Papua New Guinea type of chloroquine-resistant pfcrt in both 1994 samples and 19951996 samples. Chloroquine is the mainstay for treatment of malaria and is effective against $P$. vivax malaria in the Solomon Islands ${ }^{24}$ and Vanuatu. ${ }^{42}$ Thus, the persistence of chloroquine pressure may be the cause of the monomorphic prevalence of chloroquine-resistant $p f c r t$ in the southwestern Pacific.

In conclusion, the present study presents evidence that allelic diversity of $P$. falciparum $m s p 1$ as measured by $m s p 1$ haplotypes is not entirely dependent on the intensity of transmission. Populations from the Solomon Islands had significantly lower diversity compared with that from Thailand with a lower level of transmission. Linkage disequilibrium in msp1 was also significantly higher in the Solomon Islands compared with Thailand. These findings indicate that frequency of recombination events in mspl is determined not only by transmission intensity but also by the number of $m s p 1$ alleles prevalent in an area and multiplicity of infections.

Received March 14, 2005. Accepted for publication August 31, 2005

Acknowledgments: We thank Dr. Akira Kaneko for useful comments, and Dr. Tetsuya Hattori for suggestions about statistical analysis. We also thank all blood donors who participated in this study.

Financial support: This work was supported by a Grant-in Aid for Scientific Research on Priority Areas from the Japanese Ministry of Education, Culture, Sports, Science and Technology (no. 14021125), and by a grant for Scientific Research (C) from the Japan Society for Promotion of Science (no. 15590377).

Authors' addresses: Naoko Sakihama and Kazuyuki Tanabe, Laboratory of Biology, Osaka Institute of Technology, 5-16-1 Ohmiya, Asahi-ku, Osaka 535-8585, Japan. Hiroshi Ohmae, Institute of Basic Medical Sciences, University of Tsukuba, 1-1 Tennodai, Tsukuba City, Ibaraki 305-8575, Japan. Bernard Bakote'e, Solomon Islands Medical Training and Research Institute, P.O. Box 349, Honiara, Solomon Islands. Masato Kawabata, International Center for Medical Research, Kobe University School of Medicine, Kusunoki-cho, Chuo-ku, Kobe 650-0017, Japan. Kenji Hirayama, Department of Molecular Immunogenetics, Institute of Tropical Medicine, Nagasaki University, 1-12-4 Sakamoto, Nagasaki 852-8523, Japan.

Reprint requests: Kazuyuki Tanabe, Laboratory of Biology, Osaka Institute of Technology, 5-16-1 Ohmiya, Asahi-ku, Osaka 535-8585, Japan, Telephone/Fax: 81-6-6954-4385, E-mail: kztanabe@ge.oit.ac.jp. 


\section{REFERENCES}

1. Renia L, Ling IT, Marussig M, Miltgen F, Holder AA, Mazier D, 1997. Immunization with a recombinant C-terminal fragment of Plasmodium yoelii merozoite surface protein 1 protects mice against homologous but not heterologous $P$. yoelii sporozoite challenge. Infect Immun 65: 4419-4423.

2. Crewther PE, Matthew ML, Flegg RH, Anders RF, 1996. Protective immune responses to apical membrane antigen 1 of Plasmodium chabaudi involve recognition of strain-specific epitopes. Infect Immun 64: 3310-3317.

3. Healer J, Murphy V, Hodder AN, Masciantonio R, Gemmill AW, Anders RF, Cowman AF, Batchelor A, 2004. Allelic polymorphisms in apical membrane antigen-1 are responsible for evasion of antibody-mediated inhibition in Plasmodium falciparum. Mol Microbiol 52: 159-168.

4. Babiker HA, Lines J, Hill WG, Walliker D, 1997. Population structure of Plasmodium falciparum in villages with different malaria endemicity in east Africa. Am J Trop Med Hyg 56: 141-147.

5. Konate L, Zwetyenga J, Rogier C, Bischoff E, Fontenille D, Tall A, Spiegel A, Trape JF, Mercereau-Puijalon O, 1999. Variation of Plasmodium falciparum msp1 block 2 and msp2 allele prevalence and of infection complexicity in two neighbouring Senegalese villages with different transmission conditions. Trans $R$ Soc Trop Med Hyg 93: S1/21-S1/28.

6. Färnert A, Rooth I, Svensson, Snounou G, Björkman A, 1999. Complexity of Plasmodium falciparum infections is consistent over time and protects against clinical disease in Tanzanian children. J Infect Dis 179: 989-995.

7. Holder AA, Blackman MJ, 1994. What is the function of MSP1 on the malaria merozoite. Parasitol Today 10: 182-184.

8. Tanabe K, Mackay M, Goman M, Scaife JG, 1987. Allelic dimorphism in a surface antigen gene of the malaria parasite Plasmodium falciparum. J Mol Biol 195: 273-287.

9. Miller LH, Roberts T, Shahabuddin M, McCutchan TF, 1993. Analysis of genetic diversity in the Plasmodium falciparum merozoite surface protein-1 (MSP-1). Mol Biochem Parasitol 59: 1-14.

10. Conway DJ, Cavanagh DR, Tanabe K, Roper C, Mikes ZS, Sakihama N, Bojang KA, Oduola AM, Kremsner PG, Arnot DE, Greenwood BM, McBride JS, 2000. A principal target of human immunity to malaria identified by molecular population genetic and immunological analyses. Nat Med 6: 689-692.

11. Chang SP, Gibson HL, Lee-Ng CT, Barr PJ, Hui GS, 1992. A carboxyl-terminal fragment of Plasmodium falciparum gp195 expressed by a recombinant baculovirus induces antibodies that completely inhibit parasite growth. J Immunol 149: 548555 .

12. Blackman MJ, Heidrich HG, Donachie S, McBride JS, Holder AA, 1990. A single fragment of a malaria merozoite surface protein remains on the parasite during red cell invasion and is the target of invasion-inhibiting antibodies. J Exp Med 172: 379-382.

13. Kumar S, Yadava A, Keister DB, Tian JH, Ohl M, PerdueGreenfield KA, Miller LH, Kaslow DC, 1995. Immunogenicity and in vivo efficacy of recombinant Plasmodium falciparum merozoite surface protein-1 in Aotus monkeys. Mol Med 1: 325-333.

14. Egan AF, Burghaus P, Druilhe P, Holder AA, Riley E, 1999. Human antibodies to the $19 \mathrm{kDa}$ C-terminal fragment of Plasmodium falciparum merozoite surface protein 1 inhibit parasite growth in vitro. Parasite Immunol 21: 133-139.

15. Nwuba RI, Sodeinde O, Anumudu CI, Omosun YO, Odaibo AB, Holder AA, Nwagwu M, 2002. The human immune response to Plasmodium falciparum includes both antibodies that inhibit merozoite surface protein 1 secondary processing and blocking antibodies. Infect Immun 70: 5328-5331.

16. Kerr PJ, Ranford-Cartwright LC, Walliker D, 1994. Proof of intragenic recombination in Plasmodium falciparum. Mol Biochem Parasitol 66: 241-248.

17. Kaneko O, Kimura M, Kawamoto F, Ferreira MU, Tanabe K, 1997. Plasmodium falciparum: allelic variation in the merozoite surface protein 1 gene in wild isolates from southern Vietnam. Exp Parasitol 86: 45-57.
18. Sakihama N, Kimura M, Hirayama K, Kanda T, Na-Bangchang K, Jongwutiwes S, Conway D, Tanabe K, 1999. Allelic recombination and linkage disequilibrium within Msp1 of Plasmodium falciparum, the malignant human malaria parasite. Gene 230: $47-54$.

19. Sakihama N, Kaneko A, Hattori T, Tanabe K, 2001. Limited recombination events in merozoite surface protein-1 alleles of Plasmodium falciparum on islands. Gene 279: 41-48.

20. Conway DJ, Roper C, Oduola AM, Arnot DE, Kremsner PG, Grobusch MP, Curtis CF, Greenwood BM, 1999. High recombination rate in natural populations of Plasmodium falciparum. Proc Natl Acad Sci U S A 96: 4506-4511.

21. Tanabe K, Sakihama N, Nakamura Y, Kaneko O, Kimura M, Ferreira MU, Hirayama K, 2000. Selection and genetic drift of polymorphisms within the merozoite surface protein-1 gene of Plasmodium falciparum. Gene 241: 325-331.

22. Da Silveira LA, Ribeiro WL, Kirchgatter K, Wunderlich G, Matsuoka H, Tanabe K, Ferreira MU, 2001. Sequence diversity and linkage disequilibrium within the merozoite surface protein-1 (msp-1) locus of Plasmodium falciparum: A longitudinal study in Brazil. J Eukaryot Microbiol 48: 433-439.

23. Mehlotra RK, Fujioka H, Roepe PD, Janneh O, Ursos LMB, Jacobs-Lorena V, McNamara DT, Bockarie MJ, Kazura JW, Kyle DE, Fidock DA, Zimmerman PA, 2001. Evolution of a unique Plasmodium falciparum chloroquine-resistance phenotype in association with pfcrt polymorphism in Papua New Guinea and South America. Proc Natl Acad Sci USA 98: 12689-12694.

24. National Malaria Control Programme Annual Report, 1996. Honiara, Solomon Islands: Ministry of Health and Medical Services Government of Solomon Islands.

25. Sakihama N, Mitamura T, Kaneko A, Horii T, Tanabe K, 2001. Long PCR amplification of Plasmodium falciparum DNA extracted from filter paper blots. Exp Parasitol 97: 50-54.

26. Sakihama N, Matsuo T, Mitamura T, Horii T, Kimura M, Kawabata M, Tanabe K, 2004. Relative frequencies of polymorphisms of variation in Block 2 repeats and 5' recombinant types of Plasmodium falciparum msp1 alleles. Parasitol Int 53 : 59-67.

27. Tanabe K, Sakihama N, Färnert A, Rooth I, Björkman A, Walliker D, Ranford-Cartwright L, 2002. In vitro recombination during PCR of Plasmodium falciparum DNA: a potential pitfall in molecular population genetic analysis. Mol Biochem Parasitol 122: 211-216.

28. Qari SH, Shi Y-P, Goldman IF, Nahlen BL, Tiberenc M, Lal AA, 1998. Predicted and ovserved alleles of Plasmodium falciparum merozoite surface protein-1 (MSP-1), a poteintial vaccine antigen. Mol Biochem Parasitol 92: 241-252.

29. Fidock DA, Nomura T, Talley AK, Cooper RA, Dzekunov SM, Ferdig MT, Ursos LM, Sidhu AB, Naude B, Deitsch KW, Su XZ, Wootton JC, Roepe PD, Wellems TE, 2000. Mutations in the $P$. falciparum digestive vacuole transmembrane protein PfCRT and evidence for their role in chloroquine resistance. Mol Cell 6: 861-871.

30. Nei M, 1987. Molecular Evolutionary Genetics. New York: Colombia University Press.

31. Tanabe K, Sakihama N, Kaneko K, 2004. Stable SNPs in malaria antigen genes in isolated populations. Science 303: 493.

32. Snounou G, Zhu X, Siripoon N, Jarra W, Thaithong S, Brown KN, Viriyakosol S, 1999. Biased distribution of msp1 and msp2 allelic variants in Plasmodium falciparum populations in Thailand. Trans $R$ Soc Trop Med Hyg 93: 369-374.

33. Levinson G, Gutman GA, 1987. Slipped-strand mispairing: a major mechanism for DNA sequence evolution. Mol Biol Evol 4: 203-221.

34. Paul RE, Hackford I, Brockman A, Muller-Graf C, Price R, Luxemburger C, White NJ, Nosten F, Day KP, 1998. Transmission intensity and Plasmodium falciparum diversity on the northwestern border of Thailand. Am J Trop Med Hyg 58: 195-203.

35. Hii JLK, Kanai L, Foligela A, Kan SKP, Burkot TR, Wirtz RA, 1993. Impact of permethrin-impregnated mosquito nets compared with DDT-house-spraying against malaria transmission by Anopheles farauti and An. punctulatus in the Solomon Islands. Med Vet Entomol 7: 333-338.

36. Babiker HA, Ranford-Cartwright LC, Currie D, Charlwood JD, 
Billingsley P, Teuscher T, Walliker D, 1994. Random mating in a natural population of the malaria parasite Plasmodium falciparum. Parasitology 109: 413-421.

37. Bendixen M, Msangeni HA, Pedersen BV, Shayo D, Bodker R, 2001. Diversity of Plasmodium falciparum populations and complexity of infections in relation to transmission intensity and host age: a study from the Usambara Mountains, Tanzania. Trans R Soc Trop Med Hyg 95: 143-148.

38. Silva NS, Silveira LA, Machado RL, Povoa MM, Ferreira MU, 2000. Temporal and spatial distribution of the variants of merozoite surface protein-1 (MSP-1) in Plasmodium falciparum populations in Brazil. Ann Trop Med Parasitol 94: 675688.
39. Eisen D, Billman-Jacobe H, Marshal VF, Fryauff D, Coppel RL, 1998. Temporal variation of the merozoite surface protein-2 gene of Plasmodium falciparum. Infect Immun 66: 239-246.

40. Babiker HA, Satti G, Walliker D, 1995. Genetic changes in the population of Plasmodium falciparum in a Sudanese village over a three-year period. Am J Trop Med Hyg 53: 7-15.

41. Issifou S, Ndjikou S, Sanni A, Lekoulou F, Ntoumi F, 2001. No influence of the transmission season on genetic diversity and complexity of infections in Plasmodium falciparum isolates from Benin. Afr J Med Sci suppl 30: 17-20.

42. Kaneko A, Taleo G, Kalkoa M, Yamar S, Kobayakawa T, Björkman A, 2000. Malaria eradication on islands. Lancet 356: 15601564. 